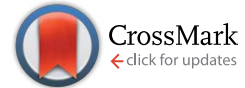

Cite this: RSC Adv., 2017, 7, 17480

Received 19th January 2017 Accepted 7th March 2017

DOI: $10.1039 / \mathrm{c} 7 \mathrm{ra00815e}$

rsc.li/rsc-advances

\section{Exhaled isopropanol: new potential biomarker in diabetic breathomics and its metabolic correlations with acetone}

\begin{abstract}
Wenwen Li, ${ }^{a}$ Yong Liu, ${ }^{a}$ Yu Liu, ${ }^{b}$ Shouquan Cheng ${ }^{c}$ and Yixiang Duan*d
Concomitant findings of acetone (ACE) and isopropanol (IPA) in blood and other biological samples have been reported in diabetic decedents and clinic cases, but exhaled IPA has rarely been studied in breath research. This study aimed to investigate expression of exhaled IPA in diabetes and further explore the correlations between exhaled IPA and ACE, and to evaluate diabetes diagnostic applicability of exhaled IPA in combination with ACE. Exhaled breath samples at one time point from 85 type 2 diabetic patients and 56 healthy controls, and from four healthy individuals after ketogenic diet experiments were analyzed by gas chromatography mass spectrometry coupled with solid phase micro-extraction technique. Concentrations of exhaled IPA in the diabetic group (mean 85.44 ppbv) were significantly higher than those in the healthy group (mean 17.99 ppbv, $p<0.001$ ). Ketogenic diet experiments showed that both IPA and ACE levels were elevated after keto-meals when under fat-consuming metabolic states. And they shared a similar changing pace, even though there was no linear relationship between IPA and ACE in terms of concentrations. The Spearman Correlation Coefficient between exhaled IPA and ACE in diabetes was 0.66, which indicated that IPA and ACE were metabolically correlated. ROC curve analysis showed that IPA possessed promising discriminatory ability (AUC 0.86) with a sensitivity of $75.3 \%$ and a specificity of $85.7 \%$ for diabetes diagnosis. This research indicates that endogenously produced IPA is a valuable biomarker for noninvasive diabetes diagnosis in breathomics analysis. This work also addressed the speculation that IPA can be metabolized from ACE via the reversible action of alcohol dehydrogenase.
\end{abstract}

\section{Introduction}

Breath analysis has gained attention in recent decades in biochemical and molecular biology fields. ${ }^{\mathbf{1} 2}$ In the clinic, breath analysis has considerable potential for nonintrusive diagnosis of disease. ${ }^{3}$ Breathomics refers to the metabolomic study of exhaled breath and focuses on health-related breath biomarkers. ${ }^{4}$ The correlation between exhaled breath and diabetes comes from the "fruity" smell described in ancient medicine, although breath biomarkers for diabetes vary in different research reports. Exhaled acetone (CAS: 67-64-1, ACE), a known biomarker with great potential for diabetes diagnosis, has been correlated with diabetes since the beginning of breath analysis. The concentrations and metabolic mechanisms of

${ }^{a}$ Research Center of Analytical Instrumentation, Analytical and Testing Center, Sichuan University, Chengdu, P. R. China

${ }^{b}$ Department of Endocrinology, The Second Hospital of Jilin University, Changchun, P. R. China

'208 Hospital of People's Liberation Army, Changchun, P. R. China

${ }^{d}$ Research Center of Analytical Instrumentation, Key Laboratory of Bio-resource and Eco-environment, Ministry of Education, College of Life Science, Sichuan University, 29 Wangijang Road, Chengdu 610064, P. R. China. E-mail: yduan@scu.edu.cn; Fax: +8628 85418180; Tel: +862885418180
ACE in patients with diabetes have been investigated in a number of studies. ${ }^{5-7}$ ACE has also been used to predict diabetic blood glucose $\mathrm{e}^{\mathbf{8 , 9}}$ and to monitor diabetic ketoacidosis. ${ }^{10,11}$ Isopropanol (CAS: 67-63-0, IPA) and ACE have been reported in biological fluids including blood, urine, and vitreous humor from patients with diabetes mellitus and diabetic ketoacidosis. ${ }^{12-15}$ And IPA was postulated to be a reduction product of ACE through reverse reaction of alcohol dehydrogenase $(\mathrm{ADH}){ }^{16}$

The broad utility and high volatility of IPA mean that IPA present in deceased biological fluids was often considered to be IPA poisoning via oral ingestion, transdermal absorption, and/ or inhalation. ${ }^{17-20}$ However, there have been some reports of IPA detection without any suspicion of IPA contamination. Serum IPA was found in an alcohol ketoacidosis patient who was rid of IPA exposure. ${ }^{21}$ Molina et al. found detectable IPA concentrations in decedents with diabetes mellitus, infection, and/or chronic ethanol abuse. ${ }^{14}$ This led to speculation that the endogenous IPA resulted from ACE metabolism. Usually, IPA is metabolized to ACE by ADH in the liver after ingestion, ${ }^{22}$ which was considered to be the reason for coexistence of ACE and IPA in body fluids of decedents. For cases with histories of no exposure to IPA, researchers conjectured that the presence of 
IPA in biological fluids may not represent acute ingestion, but rather reverse metabolism from ACE. One of first study groups to test this speculation was Lewis et al., who reported measurable levels of IPA in the serum of rats on increasing doses of ACE. ${ }^{23}$ Robertson et al. speculated that IPA may be reduced in vivo by ACE on finding considerable amounts of IPA in the blood of acetonemic cows. ${ }^{24}$ Davis et al. further verified the hypothesis in an in vitro experiment, by incubating mixtures of $\mathrm{ACE}, \mathrm{ADH}$, and $\mathrm{NADH}$ (nicotinamide adenine dinucleotide hydrogen) at $\mathrm{pH} 7.3$ and 8.8, with results showing continuous increasing production of IPA over the test intervals. ${ }^{25}$ All these in vivo and vitro experiments indicated that the reverse reaction of $\mathrm{ADH}$ to produce IPA was favored in states of excess ACE production and high $\mathrm{NADH} / \mathrm{NAD}^{+}$ratios. Some disease states including diabetes mellitus, starvation, high fat intake, and chronic alcoholism can result in increased ACE levels and $\mathrm{NADH} / \mathrm{NAD}^{+}$ratios. $^{21,25}$ Thus, IPA was more likely found in cases with these diseases.

IPA concentration and its relation with ACE have been discussed in serum and other biological matrices (urine, vitreous humor, etc.) of patients with diabetes mellitus, diabetic ketoacidosis (DKA), alcohol ketoacidosis (AKA), and alcohol misuserelated deaths. ${ }^{26,27}$ Davis et al. found concentrations of IPA ranging from 1 to $29 \mathrm{mg} \mathrm{dL}^{-1}$ and ACE ranging from 7 to $62 \mathrm{mg}$ $\mathrm{dL}^{-1}$ in individuals with diabetes or other diseases. ${ }^{25}$ David et al. found concentrations of IPA ranging from 2 to $7.3 \mathrm{mg} \mathrm{dL}^{-1}$ and ACE concentrations of 5.8 to $32.1 \mathrm{mg} \mathrm{dL}^{-1}$ in diabetic acetonemic patients. ${ }^{13}$ Usually, endogenously producing IPA concentrations tended to be low and the ratios of IPA/ACE were less than $1.0 .^{13,15,28}$ On the contrary, IPA levels and IPA/ACE ratios were relatively high after ingestion of IPA. ${ }^{\mathbf{1 4 , 1 5 , 2 8}}$ Petersen et al. detected IPA and ACE in 260 decedents involving DKA (diabetic ketoacidosis), AKA, and IPA intoxication conditions. The results indicated that DKA presented high ACE but low IPA levels with IPA/ACE ratio < 1.0, and AKA showed low ACE and low IPA levels with IPA/ACE ratio $<1.0$. For IPA intoxication, it had high ACE and high IPA with IPA/ACE ratio $>1.0 .^{28}$ Jenkins et al. investigated IPA and ACE concentrations in heart blood, femora blood, urine, and vitreous humor for 162 cases without IPA exposure. The results indicated that IPA levels from endogenous formation were low, and IPA/ACE ratios were typically less than $1.0 .^{15}$

Exhaled IPA has been found in exhaled breath of healthy individuals. ${ }^{29,30}$ And in a study by Yan et al. ${ }^{31}$ exhaled IPA was selected as a biomarker for diabetes diagnosis in a multivariate model. A systematic study for exhaled IPA is lacking, but exploration of exhaled IPA and its metabolic relation with ACE is worthwhile in diabetic breathomics for noninvasive diagnosis. In this study, exhaled IPA concentrations at one time point from type 2 diabetic patients and healthy subjects were measured by gas chromatography mass spectrometry (GC/MS) coupled with solid phase micro-extraction (SPME) technique. Correlation between IPA and ACE was investigated in diabetes and healthy groups. Ketogenic diet experiments on healthy individuals were developed to explore the in vivo metabolic relations between ACE and IPA under a fat consuming process. The study aimed to clarify correlations between biomarkers in diabetic breathomics, thus promoting breath-analysis-based disease diagnosis.

\section{Subjects and methods}

\section{Diabetes subjects and controls}

The study population were all Asian and all were volunteers. The study protocol was approved by the medical ethics committee of the PLA 208 Hospital (Jilin, China), in accordance with the 2013 Declaration of Helsinki. ${ }^{32}$ Each participant was sent information on the experimental process, to give informed consent. A total of 85 type 2 diabetes (T2D) patients (41 males and 44 females, aged 20-85 years) and 56 healthy controls (24 males and 32 females, aged 21-71 years) were recruited. The diabetic subjects were newly diagnosed as type 2 diabetes according to the criteria of WHO (World Health Organization), ${ }^{33}$ and no surgical or medical treatment had been undertaken before the breath sampling. Healthy subjects were recruited from Sichuan University randomly. Based on a questionnaire for controls and admission protocols for patients, the exclusion criteria were subjects with respiratory and lung diseases; smokers and chronic alcoholics; and healthy controls suffering acute inflammation or those on a diet.

\section{Ketogenic diet experiment}

A ketogenic diet experiment on healthy subjects was implemented to investigate the metabolic correlation between ACE and IPA. A ketogenic diet was defined as low-carbohydrate, adequate-protein, and high-fat intake. Ketogenic meals with a ketogenic ratio of $3.8: 1$ (fat : protein + carbohydrate) ${ }^{34}$ were designed using a Keto Calculator formula, ${ }^{35}$ which took factors of body weight (kg), basal metabolic rate, activity level, and fat/ carbohydrate ratio into consideration for each individual. The calculated result included total calorie intake (kcal) and separate intake $(\mathrm{g})$ for net carbohydrates, protein, and fat. Whipping cream $(35.1 \%$ fat containing, Horeca, Germany) and protein powder (ByHealth, China) were supplied for each subject based on the keto calculation result. Four healthy individuals (aged 24-30, three males and one female) in the laboratory joined in with the ketogenic experiment. Subjects were required to fast for at least $10 \mathrm{~h}$ overnight to process the deposited carbohydrates before arriving at the laboratory at 8:00 am. Concentrations of IPA and ACE had been measured the previous afternoon, and then were measured in the early morning for comparison. Then, a ketogenic meal containing whipping cream and protein powder was provided without any other intake except water. Exhaled samples were collected hourly for $4 \mathrm{~h}$ from 8:00 am to 12:00 am.

\section{Breath sample collection}

Breath samples were collected in $3 \mathrm{~L}$ multi-layer aluminum foil bags (Dalian Delin Gas Packing Co. Ltd, China), which ensured a comparatively long storage time for exhaled components. The bags were baked at $40{ }^{\circ} \mathrm{C}$ for $6 \mathrm{~h}$ and then flushed with high purity nitrogen three times before use. The gas inlet port of the bag was a three-way valve, which excluded the first 2-3 s dead 
space gases and ensured collection of the alveolar gas. The sampling process was done in a clean room with good ventilation. For diabetic patients, the breath samples were collected on admission to hospital, and meanwhile, biochemical tests for blood ketone and urine ketone were implemented at the hospital. Subjects with possible ambient IPA or ACE (and any other VOCs) exposure, long-term smokers, and those abusing alcohol were excluded from the study, thus avoiding any exogenous contamination and guaranteeing the endogenous generation of IPA. All subjects were required to fast overnight prior to breath sampling. Physical exercise was restrained to avoid any metabolism variation. Participants had $10 \mathrm{~min}$ rest in the sampling room for gas exchange to occur before sampling. Subjects were required to exhale a single breath gently until mild resistance was felt, and some practice was taken before sampling. Background air was collected and analyzed in parallel for diabetes patients and controls. Background correction was implemented by subtracting ambient IPA and ACE from the exhaled samples. Breath samples were collected and then sent to the laboratory. Samples were analyzed immediately on their arrival day.

\section{SPME and GC/MS procedure}

Breath samples in this work were analyzed using a 7890A GC coupled to a 220 ion trap MS (Agilent, USA). A DB-624 fused-silica capillary column $(60 \mathrm{~m} \times 0.25 \mathrm{~mm}$ diam. $\times 1.40 \mu \mathrm{m}$ films, Agilent, USA) was used to separate the exhaled volatile organic compounds (VOCs). Breath samples were preconcentrated using an $85 \mu \mathrm{m}$ Car/PDMS fiber (Sigma-Aldrich, USA) for $35 \mathrm{~min}$ at room temperature $\left(20^{\circ} \mathrm{C}\right)$. Then the fiber was inserted into the GC front inlet for thermal desorption at $250{ }^{\circ} \mathrm{C}$ for $5 \mathrm{~min}$. The GC temperature programming was set as follows: initially at $40{ }^{\circ} \mathrm{C}$ for $5 \mathrm{~min}$, increasing to $160{ }^{\circ} \mathrm{C}$ at a rate of $10^{\circ} \mathrm{C} \mathrm{min}{ }^{-1}$, and then increasing to $200^{\circ} \mathrm{C}$ at $5{ }^{\circ} \mathrm{C} \mathrm{min}^{-1}$ and maintained for $5 \mathrm{~min}$. The split ratio was $10: 1$ at the GC front inlet. The carrier gas was helium with a flow rate of $1.0 \mathrm{~mL} \mathrm{~min}^{-1}$. The MS detector was under full scan mode with $\mathrm{m} / \mathrm{z}$ range from 40 to 300 and a scan rate of 0.5 scans per s. Temperature was set at $220^{\circ} \mathrm{C}, 120^{\circ} \mathrm{C}$, and $250{ }^{\circ} \mathrm{C}$ for trap, manifold, and xferline, respectively.

\section{Method validation and statistical analyses}

Raw data from GC/MS were analyzed using Workstation Toolbar, version 6.9.3 (Varian). Peak areas of IPA and ACE were obtained by integrating the selected ion chromatography of $45 \mathrm{~m} / \mathrm{z}$, and 43 and $58 \mathrm{~m} / \mathrm{z}$, respectively. A calibration curve of peak areas $(Y)$ and IPA concentrations $(x, \mathrm{ppbv})$ was $Y=554 x+16169\left(R^{2}=0.99\right)$, which covered a linear range from $9.8 \mathrm{ppbv}$ to $1261 \mathrm{ppbv}$. The IPA gas standards with concentrations of 9.8, 21, 42, 210, 420, 841 , and 1216 ppbv were prepared by injecting $4 \mu \mathrm{L}$ of stocked relevant IPA solutions into the aluminum foil bags. Limit of detection (LOD) was $0.51 \mathrm{ppbv}$, which was estimated based on three times signal-to-noise ratio from a calibration sample at a low concentration. Limit of quantitative (LOQ) was 1.69 ppbv based on ten times signal-to-noise ratio. The accuracy for IPA measurement was estimated by the recovery (94-108\%) through measurement for a standard concentration in quintuplicate. Method validation for ACE was done in a previous work. ${ }^{7}$ The statistical analyses were performed by SPSS 20.0 (SPSS Inc., USA) and SIMCA 13.0 (Umetrics, Sweden). Nonparametric MannWhitney $U$ test was to detect significant differences in the distributions between two groups. Spearman Rank Correlation analysis, a nonparametric statistic method, was used to evaluate the correlations for non-normal distribution data. The receiver operating characteristic (ROC) curve and area under the curve (AUC) were used to evaluate the diagnostic values of biomarkers.

\section{Results and discussion}

\section{Exhaled IPA concentrations in diabetes patients and controls}

Exhaled IPA concentrations for diabetes patients and controls are shown in Fig. 1. The median of exhaled IPA for 85 diabetes patients is $44.68 \mathrm{ppbv}$ (range: $8.44-781.62 \mathrm{ppbv}$ ), which is higher than that for the 46 healthy controls, $11.59 \mathrm{ppbv}$ (range: 6.36-61.53 ppbv). Ten healthy subjects had undetectable IPA levels in this study. The Mann-Whitney $U$ test was used to conduct a comparison of IPA between the diabetic and healthy groups, with $p<0.001$ indicating a significant difference of IPA concentrations between the groups. Scatters in Fig. 1(a) indicate that IPA concentrations had greater within-group variation in diabetes patients, compared with healthy controls. This is because the healthy individuals shared similar and stable metabolism status, while the diabetic patients underwent differing and aggravated IPA production. Fig. 1(b) shows some overlap of IPA concentrations between the T2D group and the healthy group. The distribution of IPA in the diabetic group is wider and shows a shift towards higher concentrations compared with the healthy group. The overlap is excusable as the type 2 diabetes was insulin-independent and some wellcontrolled patients shared a similar metabolic state to the healthy subjects in some circumstances.

In diabetes lipolysis is used for energy supply, and during this process fatty acids are promptly mobilized in, and released from, the adipose tissue. Parallel to this phenomenon, fatty acid synthesis is repressed and ketone body generation increases through increased beta-oxidation in the liver. ${ }^{36}$ The main ketone bodies are acetoacetate, beta-hydroxybutyrate, and acetone, and these are used as energy supply for the human body and especially for the brain. Acetoacetate is produced from acetyl-CoA during the lipolysis, then is metabolized to acetone and betahydroxybutyrate through decarboxylation and reduction, respectively. Acetoacetate and beta-hydroxybutyrate can be metabolized back to acetyl-CoA in extra-hepatic tissues, while the acetone is mainly eliminated via the urine and/or exhalation. High levels of ACE in the liver can result in increased production of IPA, especially in the presence of elevated NADH/ $\mathrm{NAD}^{+}$ratios. $^{28}$ Diabetes appears to cause intense lipolysis, which aggravates the beta-oxidation process and results in increased $\mathrm{NADH} / \mathrm{NAD}^{+}$ratios. ${ }^{36}$ Thus, IPA production is favored in diabetic conditions.

\section{Correlations between IPA and ACE}

The concentration relationship between exhaled IPA and ACE for the diabetes patients and control group are presented in 

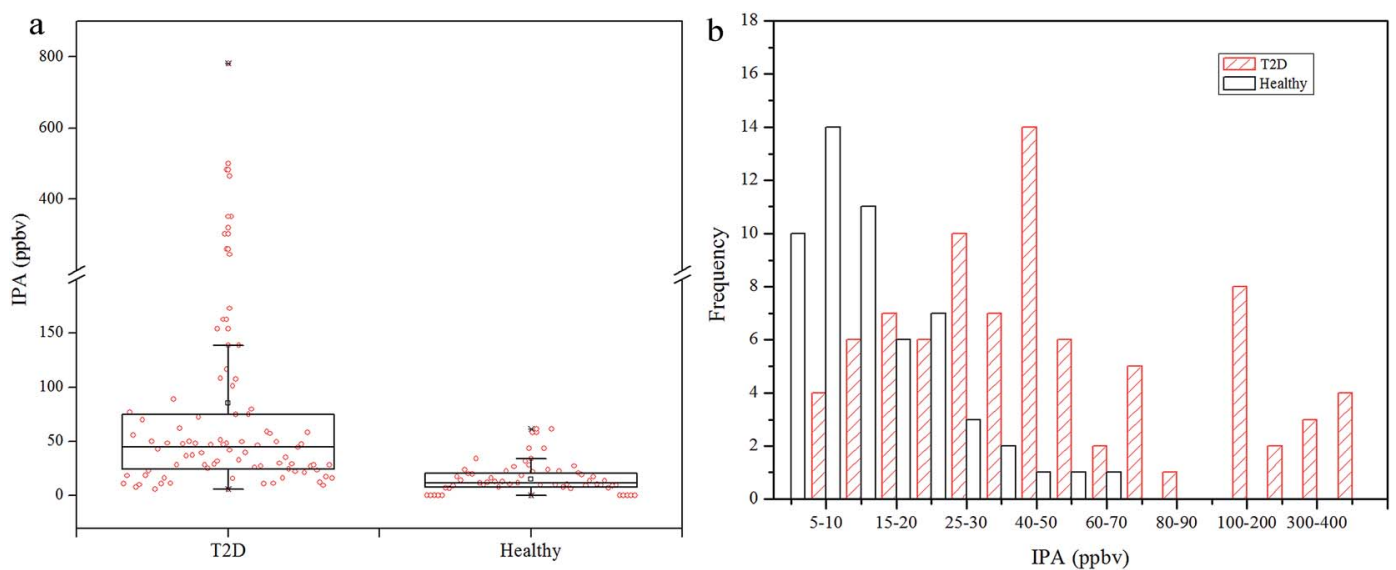

Fig. 1 (a) Box plot with data points of exhaled IPA concentrations for T2D and healthy controls. The break region on the $Y$ axis is $200-201$ ppbv. (b) Frequency distribution of exhaled IPA concentrations in T2D and healthy groups.
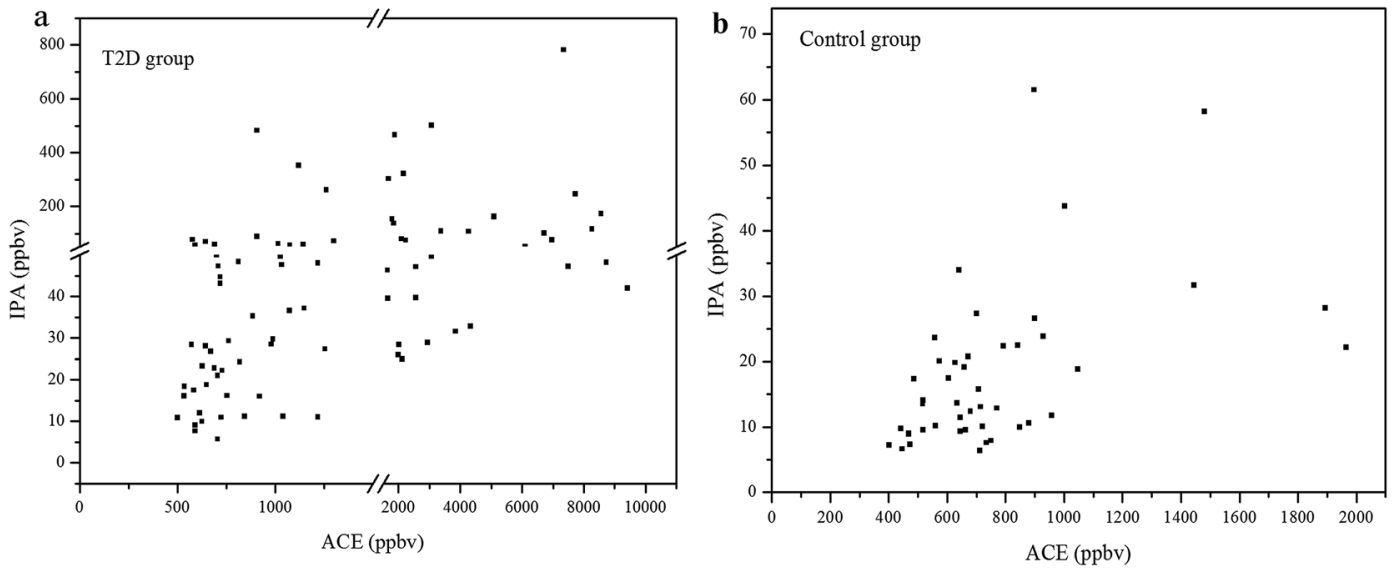

Fig. 2 (a) Concentration scatter plot of exhaled IPA versus ACE for T2D group. The break regions on the $X$ and $Y$ axes are 1500-1501 ppbv and 50-50.1 ppbv, respectively. (b) Scatter plot of exhaled IPA concentrations versus ACE for healthy control group.

Fig. 2(a) and (b), respectively. Spearman Rank Correlation analysis was used to evaluate the relationship between exhaled IPA and ACE. Exhaled ACE concentrations for diabetes patients and controls are given in Table 1. The quantitative analysis for exhaled ACE was done in a previous work. ${ }^{7}$ As can be seen in Fig. 2(a), exhaled IPA levels were found to be positively correlated with breath ACE concentrations in the diabetic group, and the Spearman Correlation Coefficient $r$ was $0.66(p<0.001)$. There was also a moderately positive correlation $(r=0.47, p=$ 0.001 ) between exhaled IPA and ACE concentrations in the control group (Fig. 2(b)). The scatter plots in Fig. 2 indicate that IPA levels had positive correlations with ACE concentrations to some extent. This is in accordance with reported speculations and research, which suggested conversion from ACE to IPA in diabetic conditions..$^{12,23}$

To explore the production extent of exhaled IPA in the diabetes and control groups, IPA/ACE ratios are discussed. The exhaled IPA/ACE ratios for the diabetic group (median 0.0319, range: $0.0045-0.5330$ ) and the healthy group (median 0.0181 , range: $0.0065-0.0686$ ) are given in the box plot in Fig. 3(a). The
IPA/ACE ratios of far less than 1.0 in this work indicate that the IPA was endogenously produced rather than exogenously ingested as reported in the literature. ${ }^{15,28}$ The frequency histogram in Fig. 3(b) indicates that the exhaled IPA presented

Table 1 Statistical summary for subjects

\begin{tabular}{|c|c|}
\hline & Mean $\pm \mathrm{SD}$ (range) \\
\hline T2D $(n=85)$ IPA (ppbv) & $85.44 \pm 129.34(8.44-781.62)$ \\
\hline $\mathrm{ACE}(\mathrm{ppbv})$ & $\begin{array}{l}2199.20 \pm 2379.89(501.76- \\
9511.94)\end{array}$ \\
\hline IPA/ACE ratio & $0.0538 \pm 0.0755(0.0045-0.5330)$ \\
\hline Blood ketone (mmol L $\left.{ }^{-1}\right)$ & $1.25 \pm 1.71(0.1-6.9)$ \\
\hline Urine ketone $\left(\mathrm{mmol} \mathrm{L}^{-1}\right)$ & $1.79 \pm 2.72(0-8)$ \\
\hline $\mathrm{FBG}\left(\mathrm{mmol} \mathrm{L}^{-1}\right)$ & $15.00 \pm 6.77(6.30-28.00)$ \\
\hline HbA1c $(\%)$ & $10.56 \pm 2.60(5.90-18.20)$ \\
\hline Healthy $^{a}(n=46)$ IPA (ppbv) & $17.99 \pm 12.21(6.36-61.53)$ \\
\hline $\mathrm{ACE}(\mathrm{ppbv})$ & $783.20 \pm 350.84(401.50-1965.60)$ \\
\hline IPA/ACE ratio & $0.0235 \pm 0.0123(0.0065-0.0686)$ \\
\hline
\end{tabular}

${ }^{a}$ There were 10 healthy subjects with undetectable isopropanol. 

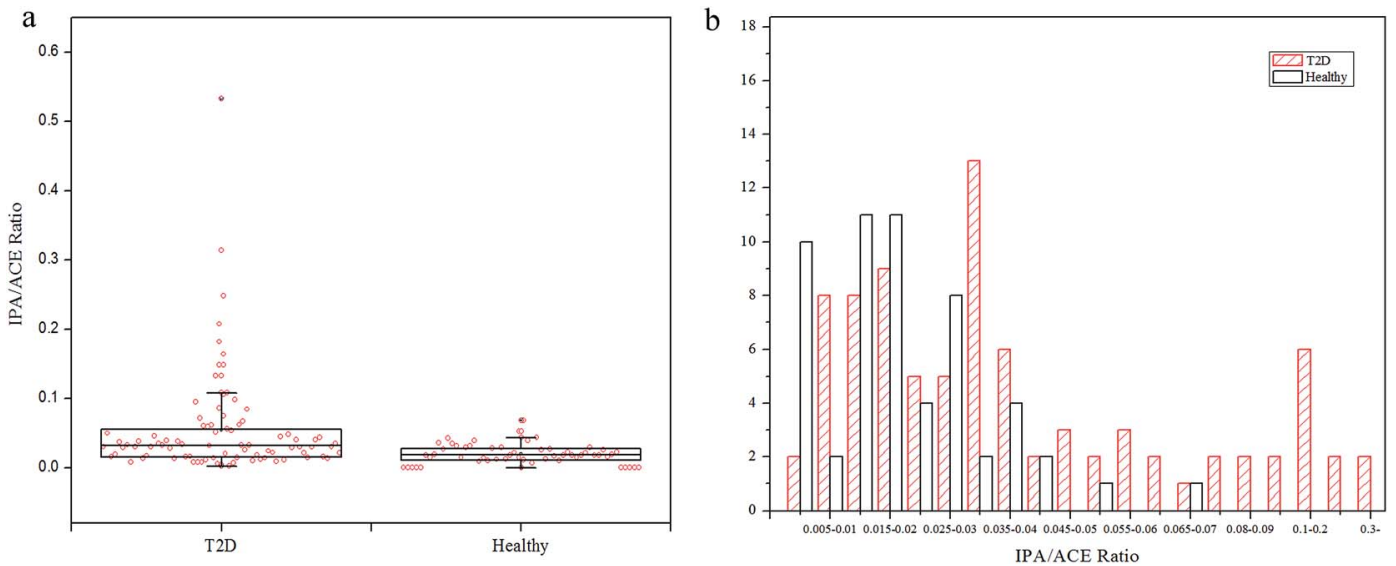

Fig. 3 (a) Box plot of IPA/ACE ratios for T2D patients and healthy controls. (b) Frequency distribution of IPA/ACE ratios in T2D and healthy groups.

a higher production rate in diabetes compared with healthy controls. In diabetic bodies, ACE is accumulated in the betaoxidation pathway during lipolysis, ${ }^{7}$ which promotes production of IPA. Additionally, $\mathrm{NADH}$, which has the capability of reducing ACE to IPA in the presence of high levels of ACE, can be generated through the beta-oxidation process. The oxidized product of $\mathrm{NADH}, \mathrm{NAD}^{+}$, in turn, can stimulate the fatty acid oxidation, thus further promoting production of ketone bodies and NADH. ${ }^{12}$ Therefore, with this increased redox state and excess ACE content, diabetes can result in a higher IPA production rate.

A ketogenic experiment was implemented on four healthy individuals to investigate the metabolic relation between IPA and ACE. Lipolysis was favored under conditions of starvation, high fat intake, diabetes mellitus, and alcohol abuse through the beta-oxidation pathway. During this process, excess acetone and increased $\mathrm{NADH} / \mathrm{NAD}^{+}$ratios were stored in the liver, and these are key materials for isopropanol production. The fatconsuming metabolism process in healthy bodies supplied with keto meals was used to mimic the lipolysis process in diabetic bodies. Three males and one female colleague in the laboratory were involved in the ketogenic diet experiment. Fig. 4 shows the metabolic changes in IPA and the simultaneous ACE concentrations after the ketogenic meal. The graphs on the left in Fig. 4 show concentrations of IPA and ACE with double $Y$ axis, and the graphs on the right show the corresponding IPA/ ACE ratios. For comparison, four time-point measurements of exhaled IPA and ACE concentrations under normal conditions were made in the afternoon (14:00-17:00) before experiment, and early morning (8:00) breath samples were also collected as baseline. As can be seen in the left panel of Fig. 4, under normal metabolic status (from 14:00 to 17:00), exhaled IPA and ACE concentrations remained stable in all subjects. Early morning exhaled ACE and IPA concentrations were elevated after $10 \mathrm{~h}$ overnight fasting compared with those measured in the afternoon. After the baseline sampling, ketogenic meals consisting of calculated whipping cream and protein powder were provided to subjects. Increased ACE concentrations after ketogenic meals were clearly observed for all subjects, but the metabolic changes did not share a similar trend for different individuals. Meanwhile, exhaled IPA levels were also elevated after ketogenic meals for all subjects with differing metabolic tendencies. Interestingly, after the ketogenic meals, the exhaled IPA displayed a similar changing trend for subjects (a) and (c), who possessed high IPA concentrations of more than $100 \mathrm{ppbv}$, and analogous changes occurred in subjects (e) and (f) who produced comparatively low IPA concentrations. Spearman correlation analysis was also used here to evaluate the metabolic correlation between IPA and ACE. The correlation coefficients for subjects (a), (c), (e), and (g) were $0.85(p=0.004), 0.78$ $(p=0.013), 0.78(p=0.013)$, and $1.00(p<0.001)$, respectively. IPA production was elevated along with the aggravated ACE metabolism during the lipolysis process after the ketogenic diet, even though the changes for exhaled IPA levels did not closely follow those for the simultaneous breath ACE levels. The complexity of metabolism means that reduction of ACE to IPA in vivo may be influenced by the time-varying redox state and physical conditions of individuals. Therefore, in this study, there was no linear relationship between IPA and ACE in terms of concentrations. Nevertheless, IPA and ACE levels were elevated after ketogenic meals in all subjects, indicating that a fat-burning (for energy supply) body enhanced not only production of ACE but also IPA levels. The corresponding IPA/ ACE ratios are shown in the right panel of Fig. 4. In general, IPA/ACE ratios after ketogenic meals were higher than those under normal conditions, demonstrating that the IPA generation rate was elevated under the fat-consuming metabolic process.

This study, for the first time, conducted a systematic investigation into exhaled IPA. The detectable IPA concentrations in exhaled breath from diabetes patients and controls without IPA contamination addresses the possibility of endogenous production of IPA. And, also for the first time, such endogenously produced IPA was explored in human subjects using a keto-diet experiment after the in vitro experiment ${ }^{25}$ and animal testing ${ }^{23}$ reported in the literature. The ketogenic experiment showed that IPA concentrations increased considerably after metabolic stimulation, which 

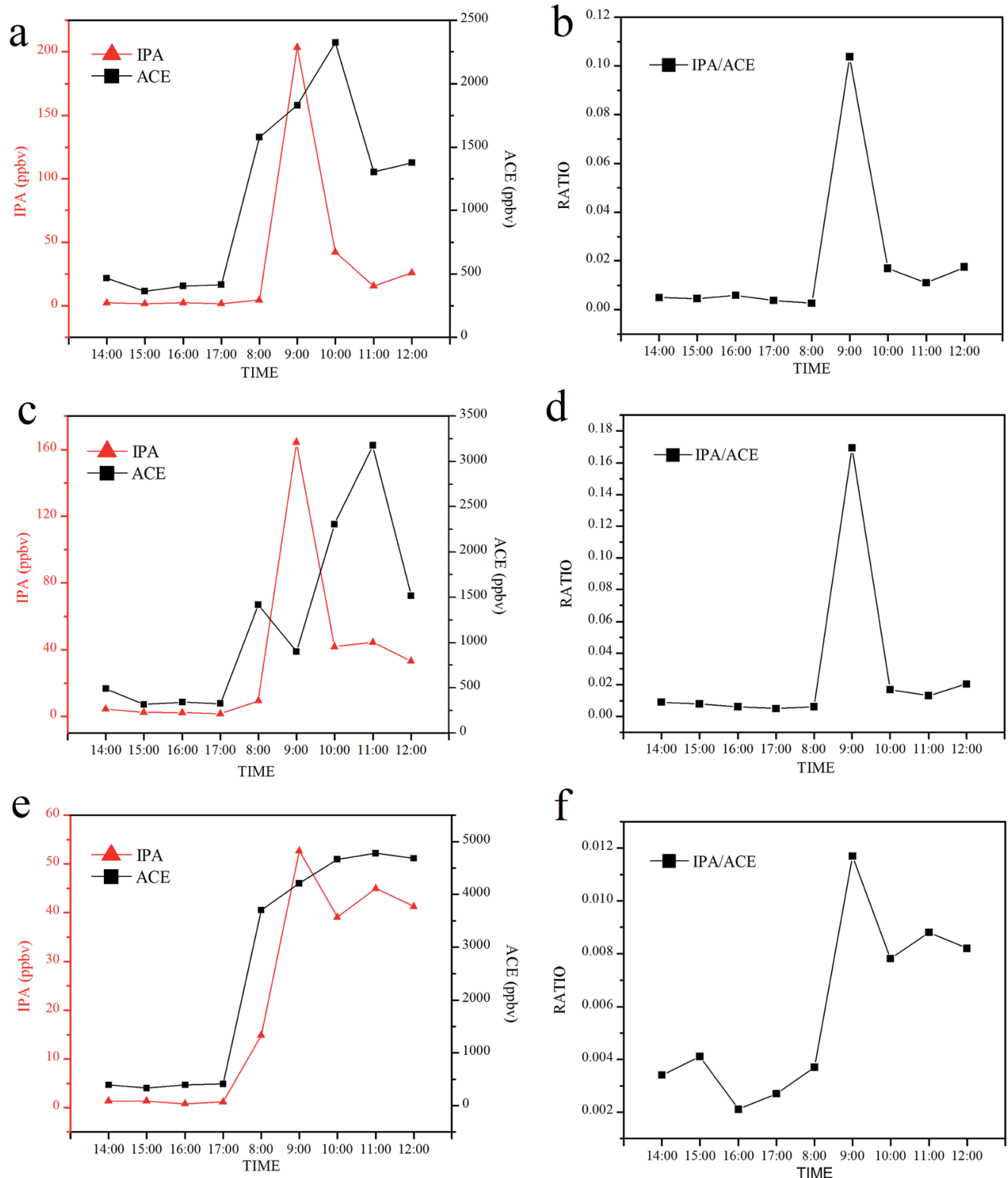

f
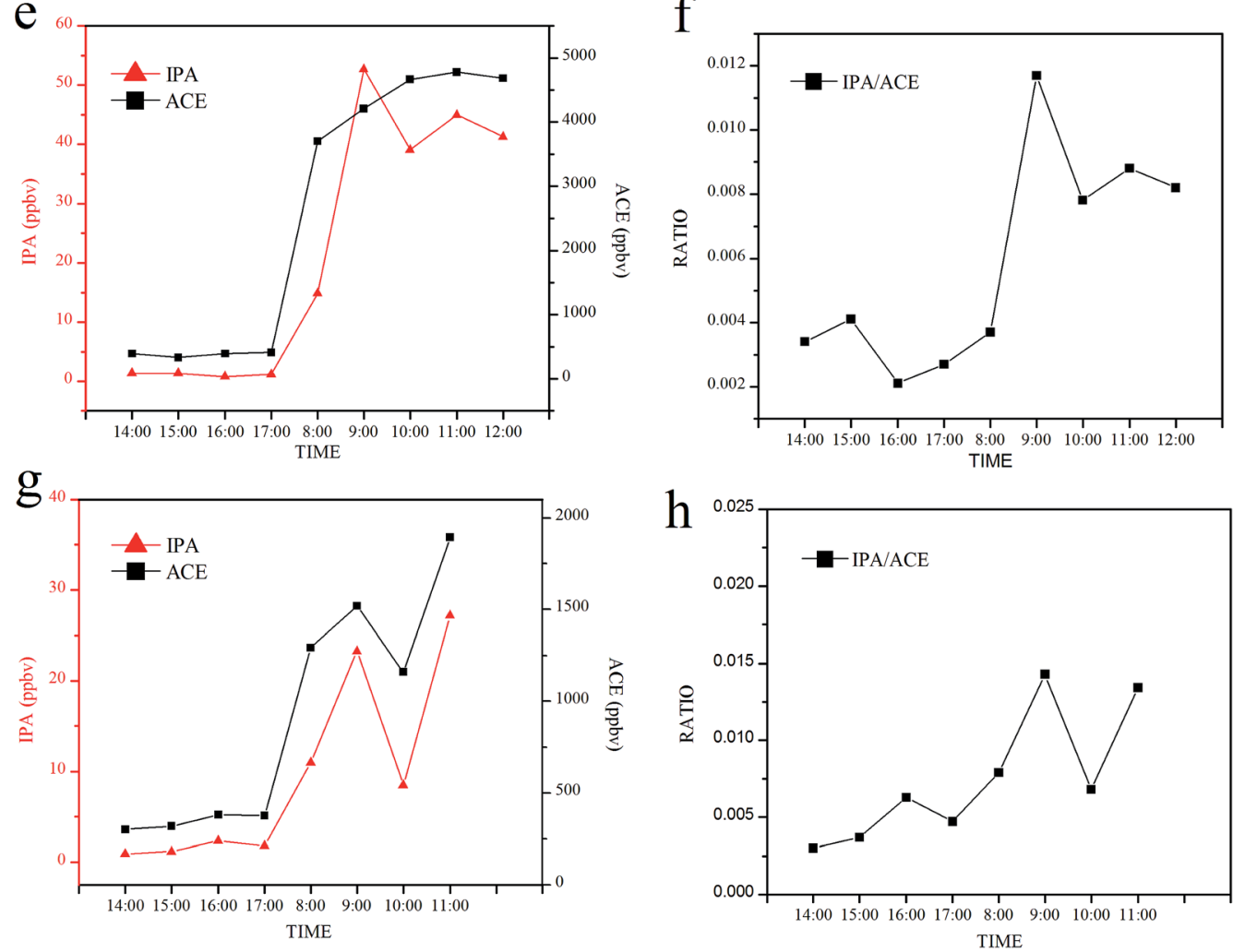

Fig. 4 Ketogenic diet experiments on four healthy individuals. IPA and ACE concentrations on the previous afternoon (14:00-17:00) and in the early morning (8:00) were measured as comparison. IPA and ACE concentrations after ketogenic meals were detected hourly for $4 \mathrm{~h}$. Graphs on the left panel (a), (c), (e), and (g) show the paired concentrations of IPA and ACE. Corresponding IPA/ACE ratios are displayed on the right side (b), (d), (f), and (h), respectively. 
further proved that IPA can be generated endogenously. The elevated exhaled IPA levels in diabetes subjects indicates that IPA is a promising biomarker for noninvasive diagnosis of diabetes. It had been suggested that IPA was generated from reduction of $\mathrm{ACE}$ through $\mathrm{ADH}$ and a high $\mathrm{NADH} / \mathrm{NAD}^{+}$ratio. The present keto-diet experiment indicated that production of IPA was closely related to ACE metabolism during the betaoxidation process. Hence exhaled IPA could be a valuable biomarker for diabetic lipolysis metabolism together with ACE in breathomics analysis.

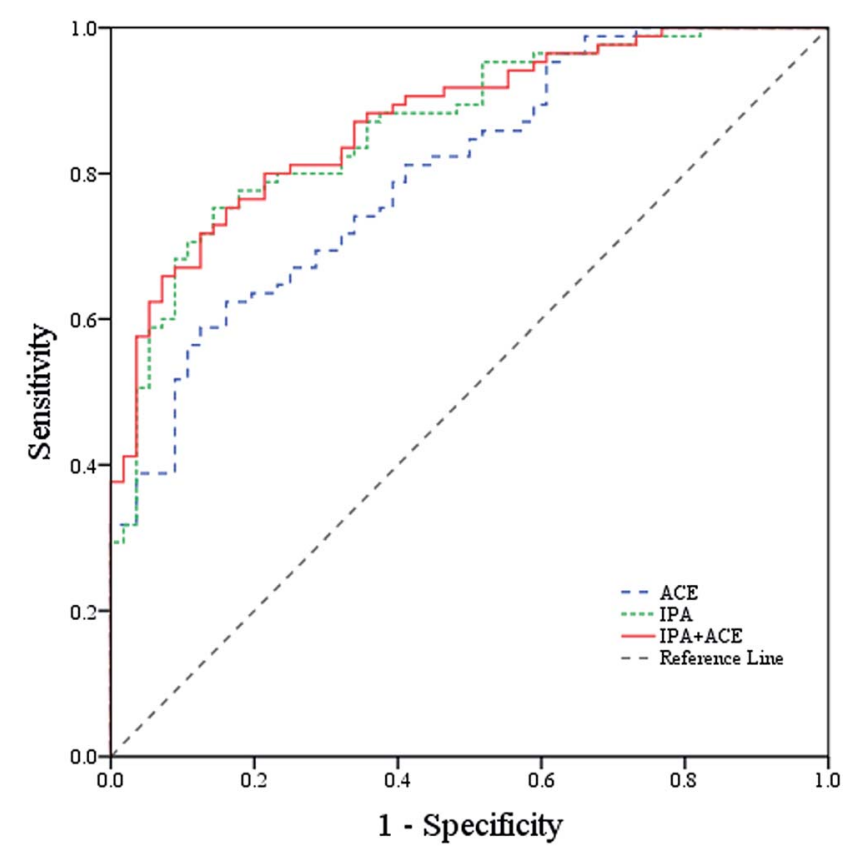

Fig. 5 Receiver operating characteristic (ROC) curve analysis for IPA, $\mathrm{ACE}$, and a combination model of IPA and ACE for differential T2D from healthy controls.

\section{Diagnostic capability of exhaled IPA for diabetes}

The ROC curves in Fig. 5 show that breath ACE possessed fair discriminatory value (AUC 0.80, 95\% CI: $0.73-0.87, p<0.001$ ) with a sensitivity of $62.4 \%$ and a specificity of $83.9 \%$. ACE concentrations for T2D and healthy subjects were measured in a previous work, and a statistical summary is given in Table 1. Exhaled IPA provided improved discriminatory ability (AUC $0.86,95 \%$ CI: $0.80-0.92, P<0.001)$ with a sensitivity of $75.3 \%$ and a specificity of $85.7 \%$. However, a combination model of the two biomarkers afforded only ordinary discriminatory ability (AUC $0.86,95 \%$ CI: $0.82-0.93, p<0.001$ ) with a sensitivity of $71.8 \%$ and a specificity of $87.5 \%$. The optimal cutoff thresholds of ACE and IPA for diabetes diagnosis were $902.99 \mathrm{ppbv}$ and 24.07 ppbv, respectively. In diabetic conditions, IPA is suggested to be a by-product of ACE, generated in the liver during beta-oxidation of fatty acid, thus increasing IPA levels may indicate a severe metabolic state of diabetes. Therefore, IPA affords better diagnostic value compared with ACE.

To explore possible relations between IPA and diabetic ketone bodies, the box plots in Fig. 6 display varying concentrations of IPA with different levels of blood ketone (betahydroxybutyrate) and urine ketone. Blood ketone (1.25 \pm $\left.1.71 \mathrm{mmol} \mathrm{L}^{-1}\right)$ and urine ketone $\left(2.79 \pm 2.72 \mathrm{mmol} \mathrm{L}^{-1}\right)$ for all diabetic patients were tested in the hospital. Urine ketone was tested using Bayer Ketostix reagent strips. Blood ketone was obtained by detecting beta-hydroxybutyrate concentration in fresh fingertip blood samples using an Optium Xceed (Abbott, USA) device, with the manufacturer-suggested cutoff of $>0.5 \mathrm{mmol} \mathrm{L}^{-1}$ used as 'positive'. Normally, ketone bodies (acetoacetate, beta-hydroxybutyrate, and ACE) produced in the liver during beta-oxidation of fatty acid act as energy sources. Diabetes appears to cause intense lipolysis, thus generating redundant ketone bodies. Relationships between breath ACE and blood ketone and urine ketone were investigated in a previous work, ${ }^{7}$ which showed that exhaled ACE positively
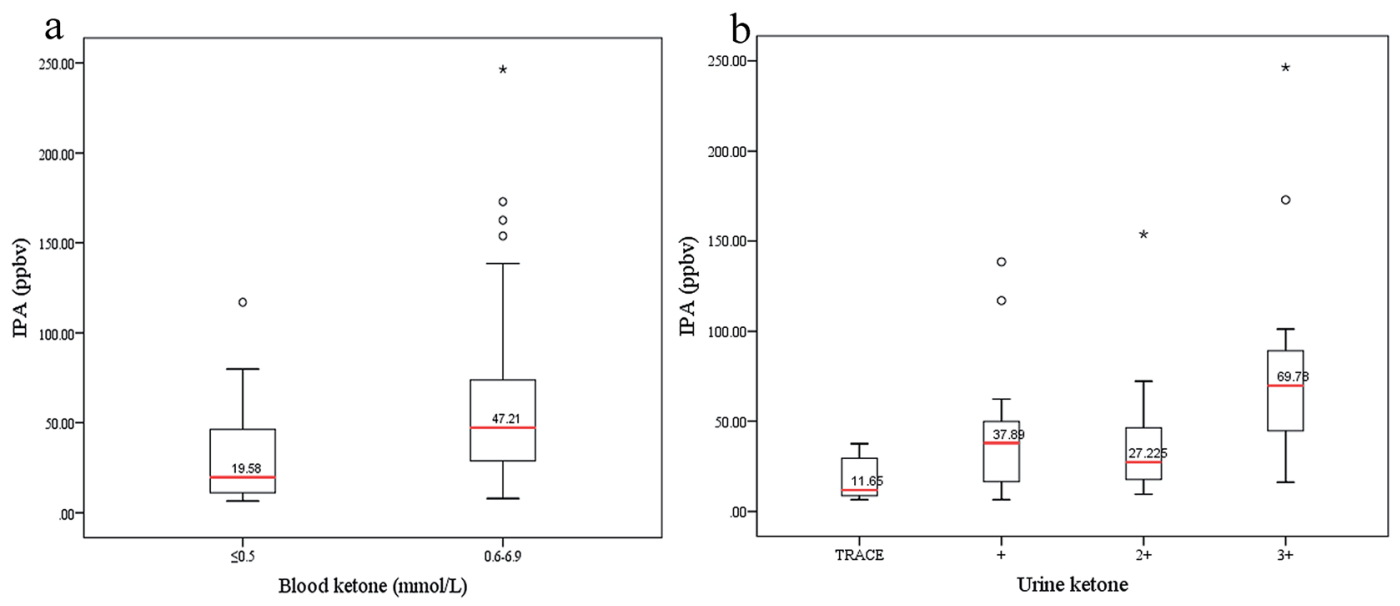

Fig. 6 (a) IPA was divided into two subgroups according to the simultaneous blood ketone values, a physiological state group with blood ketone $\leq 0.5 \mathrm{mmol} \mathrm{L}^{-1}$ and a risky group with blood ketone ranging $0.6-6.9 \mathrm{mmol} \mathrm{L}^{-1}$. (b) IPA concentrations were grouped based on different urine ketone levels. Urine ketone was tested by Bayer Ketostix reagent strips which indicated color changes of,,,$- \pm+ 2+$ and $3+$ corresponding to levels of negative, trace $\left.(0.5 \mathrm{mmol} \mathrm{L})^{-1}\right)$, mild $\left.(1.5 \mathrm{mmol} \mathrm{L})^{-1}\right)$, moderate $(4 \mathrm{mmol} \mathrm{L})$, and high $\left(8 \mathrm{mmol} \mathrm{L}^{-1}\right)$, respectively. The trace group included - and \pm levels. 
correlated with blood and urine ketones. In this study, IPA levels also increased with rising blood and urine ketones. In Fig. 6(a), blood ketone levels are divided into two subgroups, a physiological state group (ith rimmol $\mathrm{L}^{-1}$ ) and a risky group (0.6-6.9 $\left.\mathrm{mmol} \mathrm{L}^{-1}\right)$. IPA levels in the risky group (median 47.21 ppbv) are relatively higher than those in the physiological group (median 19.58 ppbv). In Fig. 6(b), urine ketones are divided into four subgroups. IPA displays obviously high levels in the fourth group with high urine ketones (median 69.78 ppbv), and comparatively low levels in the first group with trace urine ketones (median $11.65 \mathrm{ppbv}$ ), which indicates that production of IPA is correlated with diabetic metabolism states to some extent. However, it must be acknowledged that in this study there is not enough capability for IPA to differentiate diabetic conditions with mild and moderate urine ketones.

\section{Conclusions}

Measurable IPA levels have been found in blood and other biological samples from diabetes mellitus and DKA decedents, suggesting that IPA production is correlated with diabetes metabolism. Researchers speculated that production of IPA was involved with ACE metabolism in diabetes. This study examined exhaled IPA and ACE in live diabetes patients and controls. Results revealed that exhaled IPA was a promising biomarker for diabetes diagnosis in breathomics. Exhaled IPA and ACE showed a positive correlation in both the diabetes and control groups, and IPA/ACE ratios far less than 1.0 indicated that endogenously producing IPA was low either in diabetes or in controls, in accordance with reports from the literature. Through a preliminary metabolic experiment, production of both exhaled IPA and ACE were found to increase when the body consumed fat for energy. The ketogenic diet experiment indicated that the exhaled concentrations of IPA did not closely follow up the changes in ACE levels. The individual physical conditions and metabolism rates may influence this rough experiment to some extent, and some more well-controlled experiments are needed to further clarify the metabolism pathway and impact factors in diabetes. Through this study, endogenous production of IPA in diabetes patients and controls was proven, and the metabolism correlation between IPA and ACE was explored. Eventually, the study indicated that IPA was not only present in some biological matrix but also in exhaled breath, and that endogenously produced IPA is a valid biomarker for diabetes. Further exploration of metabolism relations of exhaled biomarkers would be worthwhile in breathomics for breath-based disease diagnosis, and this study was just a preliminary attempt.

\section{Acknowledgements}

The authors are grateful to all the volunteers with diabetes and healthy volunteers who positively cooperated with this work. This project was funded by the National Natural Science Foundation of China (CN) (No. 21305095).

\section{References}

1 J. Pereira, P. Porto-Figueira, C. Cavaco, K. Taunk, S. Rapole, R. Dhakne, H. Nagarajaram and J. S. Camara, Metabolites, 2015, 5, 3-55.

2 D. Smith and P. Spanel, Analyst, 2007, 132, 390-396.

3 W. Miekisch and J. Schubert, TrAC, Trends Anal. Chem., 2006, 25, 665-673.

4 A. W. Boots, J. J. van Berkel, J. W. Dallinga, A. Smolinska, E. F. Wouters and F. J. van Schooten, J. Breath Res., 2012, 6, 027108.

5 Z. Wang and C. Wang, J. Breath Res., 2013, 7, 037109.

6 M. Storer, J. Dummer, H. Lunt, J. Scotter, F. McCartin, J. Cook, M. Swanney, D. Kendall, F. Logan and M. Epton, J. Breath Res., 2011, 5, 046011.

7 W. Li, Y. Liu, X. Lu, Y. Huang, Y. Liu, S. Cheng and Y. Duan, J. Breath Res., 2015, 9, 016005.

8 J. Lee, J. Ngo, D. Blake, S. Meinardi, A. M. Pontello, R. Newcomb and P. R. Galassetti, J. Appl. Physiol., 2009, 107, 155-160.

9 T. D. Minh, S. R. Oliver, J. Ngo, R. Flores, J. Midyett, S. Meinardi, M. K. Carlson, F. S. Rowland, D. R. Blake and P. R. Galassetti, Am. J. Physiol., 2011, 300, E1166-E1175.

10 S. S. Likhodii, K. Musa and S. C. Cunnane, Clin. Chem., 2002, 48, 115-120.

11 A. Prabhakar, A. Quach, D. Wang, H. Zhang, M. Terrera, D. Jackemeyer, X. Xian, F. Tsow, N. Tao and E. Forzanil, Global Journal of Obesity, Diabetes and Metabolic Syndrome, 2014, 1, 012-019.

12 A. E. Jones and R. L. Summers, World J. Emerg. Med., 2000, 19, 165-168.

13 N. David and D. N. Bailey, J. Toxicol., Clin. Toxicol., 1990, 28, 459-466.

14 D. K. Molina, J. Forensic Sci., 2010, 55, 998-1002.

15 A. J. Jenkins, T. C. Merrick and J. M. Oblock, J. Anal. Toxicol., 2008, 32, 719-720.

16 W. Miekisch, J. K. Schubert and G. F. Noeldge Schomburg, Clin. Chim. Acta, 2004, 347, 25-39.

17 L. Adelson, Am. J. Clin. Pathol., 1962, 38, 144-151.

18 S. Agarwal, J. Med. Soc. N. J., 1979, 76, 914-916.

19 D. R. Daniel, B. H. McAnalley and J. C. Garriott, J. Anal. Toxicol., 1981, 5, 110-112.

20 S. Ghimenti, S. Tabucchi, F. G. Bellagambi, T. Lomonaco, M. Onor, M. G. Trivella, R. Fuoco and F. Di Francesco, J. Pharm. Biomed. Anal., 2015, 106, 218-223.

21 J. B. Dwyer and K. Tamama, Clin. Chim. Acta, 2013, 415, 245-249.

22 R. Nordmann, C. Ribiere, H. Rouach, F. Beauge, Y. Giudicelli and J. Nordmann, Life Sci., 1973, 13, 919-932.

23 G. D. Lewis, A. K. Laufman, B. H. McAnalley and J. C. Garriott, J. Forensic Sci., 1984, 29, 541-549.

24 A. Robertson, C. Thin and A. M. Stirling, Nature, 1950, 166, 954.

25 P. L. Davis, L. A. Dal Cortivo and J. Maturo, J. Anal. Toxicol., 1984, 8, 209-212.

26 P. L. Platteborze, P. M. Rainey and G. S. Baird, Clin. Chem., 2011, 57, 1361-1364. 
27 C. Palmiere, F. Sporkert, D. Werner, D. Bardy, M. Augsburger and P. Mangin, Leg. Med., 2012, 14, 17-20.

28 T. H. Petersen, T. Williams, N. Nuwayhid and R. Harruff, J. Forensic Sci., 2012, 57, 674-678.

29 M. Phillips, J. Herrera, S. Krishnan, M. Zain, J. Greenberg and R. N. Cataneo, J. Chromatogr. B: Biomed. Sci. Appl., 1999, 729, 75-88.

30 M. Libardoni, P. T. Stevens, J. H. Waite and R. Sacks, J.

Chromatogr. B: Anal. Technol. Biomed. Life Sci., 2006, 842, 36 M. P. Kalapos, Biochim. Biophys. Acta, 2003, 1621, $122-139$. $13-21$.
31 Y. Y. Yan, Q. H. Wang, W. W. Li, Z. J. Zhao, X. Yuan, Y. P. Huang and Y. X. Duan, RSC Adv., 2014, 4, 25430-25439.

32 World Medical Association, JAMA, J. Am. Med. Assoc., 2013, 310, 2191-2194.

33 A. D. Association, Diabetes Care, 2013, 37, S14.

34 K. Musa-Veloso, E. Rarama, F. Comeau, R. Curtis and S. Cunnane, Pediatr. Res., 2002, 52, 443-448.

35 http://www.ruled.me/keto-calculator/. 